\title{
Cluster Based Energy Efficient Topology for MANETs
}

\author{
Attar Shuaib $^{1}$, Sayyed Musaddique ${ }^{2}$, V.R. Marathe ${ }^{3}$ \\ Department of Electronics and Telecommunication Engineering, N.B. Navale Sinhgad College of Engineering and \\ Technology, Solapur, India ${ }^{1,2,3}$
}

\begin{abstract}
Due to the limited resources of the network transmission of large sized data can be a bottleneck in Adhoc network. The data delivery is done by selecting appropriate cluster heads. This kind of region diversity may be utilized to achieve higher energy efficiency. We divide the network into four regions. Node with highest energy in each region is selected as cluster head. Remaining nodes forwards their data to cluster head which buffers the data and forwards only one packet instead of all the packets received from other nodes. Therefore the overall energy consumption is reduced in such a network. In our proposed method we recalculate cluster head after every round of transmission and bypass nodes with lower energy to preserve more energy. We compare the performance with non energy centric pure region based cluster head and prove that the proposed technique fares better in terms of low energy consumption, fewer node deaths, more packet delivery ratio and improved packets par node. Here by compressing redundant data we ensure better degree of compression without compromising on mean square error.
\end{abstract}

Index term: Cluster Head, MANET, Adhoc, etc.

\section{INTRODUCTION}

A Mobile Adhoc network (MANET) (sometimes called proposed protocol DBET by using AODV[8] as routing a Mobile Adhoc and actor network In practical protocol using network simulator ns2.33[1] and compared application, energy resource of node is extremely limited with AODV and AODV with SPAN.[3] The simulation in Mobile Adhoc Network. Global network energy studies revealed that the proposed scheme perform better unbalance, we divide the large-scale network into in terms of energy, delay, and delivery ratio. In general equivalent layers and propose a Energy Conserving network topology is controlled by keeping small number Mobile Adhoc Network With Packet Buffering. Balancing Energy Consumption to Maximize Network Lifetime in Data-Gathering Adhoc Networks Involving Compressive Transmitting Theory, the novel algorithm obviously has great advantages in reducing energy consumption during transmission, prolonging network life-time and eliminating information redundancy which could realize load balance for multi-layer Mobile Adhoc network. The project is simulated in NS2 and it is proved that by using the proposed technique, network lifetime can be improved and packet delivery ratio is increased

Various techniques, in network layer, are proposed in the literature to conserve energy. These techniques can be classified mainly into two categories: by controlling the number of nodes with the smaller link cost. In the first method a small number of nodes awake to maintain the network connectivity and remaining nodes go into sleep state to conserve energy. This method is effective in low traffic conditions, because the power consumption to keep nodes awake dominates the power consumption in data transfer .In the second method, topology is controlled by keeping lesser cost links in the network.

This method is effective in high data traffic because power consumption in data transfer dominates the power required to keep nodes awake. We combine the advantages of these two techniques to dynamically adjust network topology for various network traffic conditions.

Here we present a demand based energy efficient topology (DBET) that dynamically adjust network topology for various network traffic conditions. We have simulated our of nodes awake as in the first technique. The proposed DBET keeps more number of nodes along the bulk data transfer path to conserve energy by keeping low link cost as in the second technique

\section{LITERATURE REVIEW}

We briefly describe various techniques related to our work topology control. Different topologies have been proposed in the literature to reduce the energy consumption. These methods can be classified into centralized controlling and distributed computing methods. Ideally, for mobile ad hoc network a topology should be computed and maintained in distributed asynchronous and localized manner.

$\mathrm{Li}$ and Wan [6] described a distributed protocol to construct a minimum power topology and developed an algorithm which directly finds a path whose length is within a constant factor of the shortest path. The length of the path is measured in term of energy consumption. This proposed algorithm used only local information.

A topology based on minimum spanning tree, called localized minimum spanning tree (LMST) was proposed by $\mathrm{Li}$ etal[5].. It is a localized distributed protocol with the following properties:

(1) The protocol generates a strongly connected communication graph;

(2) The degree of any node is at most six,

(3) The topology can be made symmetric by removing asymmetric links without impairing connectivity.

A simple localized distributed topological control algorithm XTC [12] is proposed by Wattenhpfer et al. 
Initially each node $u$ computes a total order $(<u)$ over all Also parallel transmission of the data helps in reducing its neighbors in the network graph $\mathrm{G}$ with respect to load in a single path and also protects the data from decreasing link quality, such as signal attenuation, and intrusion.

packet arrival rate. Then, each node start exchanges the Therefore in this work we propose a parallel data neighbor order information among all neighbors. At the transmission by suitably aggregating data for data later point, each node locally selects those neighboring nodes which will form its neighborhood in the resulting topology control graph, based on the previously exchanged neighbor order information. It covers three main advantages:

1) It does not assume the network graph to be a Unit Disk Graph,

2) It works on weighted network graphs,

3) It does not require availability of the node position information.

Authors improved by adding node mobility and extended XTC for mobile network.[7]

An energy efficient dynamic path is maintained to send data from source to destination for MANET is proposed in Sheu, Tu, and Hsu [10]. Due to mobility existing paths may not be energy efficient. So, each node in a data path dynamically updates the path by adjusting its transmissionpower. Each node in the networks determines its power for data transmission and control packets transmission according to the received beacon messages from its neighbors. In dynamic path optimization technique protocols dynamically select energy efficient path as per the requirement of dynamic topological changes in the network[4]

Another class of topology control protocols based on the k-nearest neighbor's graph (k-NNG). In k-NNG every node is connected to its $\mathrm{k}$ closest neighbors. The Local Information No Topology protocol (LINT) [9]try to keep the number of neighbors of a node within a low and high threshold centered around an optimal value. But the optimal value is not characterized. So the estimation of the number of neighbors can be inaccurate and connectivity is not guaranteed. The k-Neighbors (k-NEIGH)[2] protocol keeps the number of neighbors of a node equal to, or slightly below, a given value $\mathrm{k}$. It connects each node with $\mathrm{k}$-closest neighbor, instant of require the knowledge of all the neighbors. The communication graph that result is made symmetric by removing asymmetric edges, which has k-upper bound neighbors'.

Another way of reducing the power consumption is by using efficient energy path for transmitting packets. These methods choose smaller edges in their path to reduce transmission energy. Minimum energy consumption per packet can be achieved by choosing the optimal energy consumed path from source to destination. However, this technique does not take the nodes energy capacity into consideration. So some nodes may exhaust their power since energy consumption is not fair among the nodes in the network. There for the network lift time decrease

\section{III.PROBLEM STATEMENT}

There are several techniques for data transmission. But generally data transmissions are constrained by the size of the data and energy of the nodes. Hence suitable compression of the data is essential for data transmission. the energy requirement for transmission.

We also propose an energy based cluster head calculation where a cluster head is periodically elected by cooperation between the nodes in a area as a node with the maximum residual energy.

\section{SYSTEM ANALYSIS}

\section{Present System}

Data are transmitted from Mobiles to Destination using suitable encoding technique to reduce the number of bits. Examples are network coding Hauffman coding etc. Even though actual number of bits gets reduced in this transmission scenario, overall packet is not reduced. Also variable length encoding demands variable bandwidth allocation which is constrained in Adhoc environment. Dedicated routing from every node to destination adds tremendous routing overload. Therefore variants of cluster based routing are preferred. Cluster heads are needed to collect data from cluster members, calculate summery of data and forward it to destination.

This data forwarding from cluster heads to destination can also be using direct diffusion or through intermediate nodes using suitable routing technique. Therefore these cluster heads looses their energy very fast. It is important therefore to refresh the cluster heads periodically and select nodes with better residual energy as cluster heads for longevity of the Adhoc network. But current cluster based techniques like LEACH suggests that there should not be any cluster reformation during the transmission phase. Through our simulation we show that in transmissions with high rounds of data, such nodes tends to loose energy fast. Hence we need an alternative system which is discussed in the next section.

\section{Disadvantages of Present System}

OPackets are more that take more time to reach destination.

OToo many packet energy is lost.

OHence node is dies early.

\section{Proposed System}

In the proposed system, data's are acquired by source Mobiles and are forwarded to cluster heads. Cluster heads buffers data and forwards to destination. Data transmission is synchronous with $\mathrm{CH}$ monitor in the schedule of transmission. After destination receives data from all the destinations, cluster heads are recalculated for every area. If on the middle of data forwarding any clusterhead runs out of energy, it immediately triggers re cluster calculation. During the transmission process if a node looses energy, packets are dropped. MSE error is calculated as error sum of uncompressed data and compressed data. Proposed system's MSE is more than present system. But it is compensated through longer network lifetime and better energy saving. Proposed system also delivers more packets. For high volume of 
INTERNATIONAL JOURNAL OF INNOVATIVE RESEARCH IN ELECTRICAL, ELECTRONICS, INSTRUMENTATION AND CONTROL ENGINEERING Vol. 3, Issue 12, December 2015

data, because of high packet delivery ratio, we get better MSE performance.

\section{Advantages of Proposed system}

Energy Consumption is reduced

○ Number of packet is reduced saving communication cost

○ Base station gets aggregated data. Hence decision making is faster.

- Due to less number of packets, congestion is lower

○ Reduced the work load.

\section{SYSTEM DESIGN}

\subsection{INTRODUCTION}

Systems design is the method of defining the design, modules, elements, interfaces, and knowledge for a system to satisfy specified needs. Systems design can be seen because the application of systems theory to development.

\subsection{DESIGN OVERVIEW}

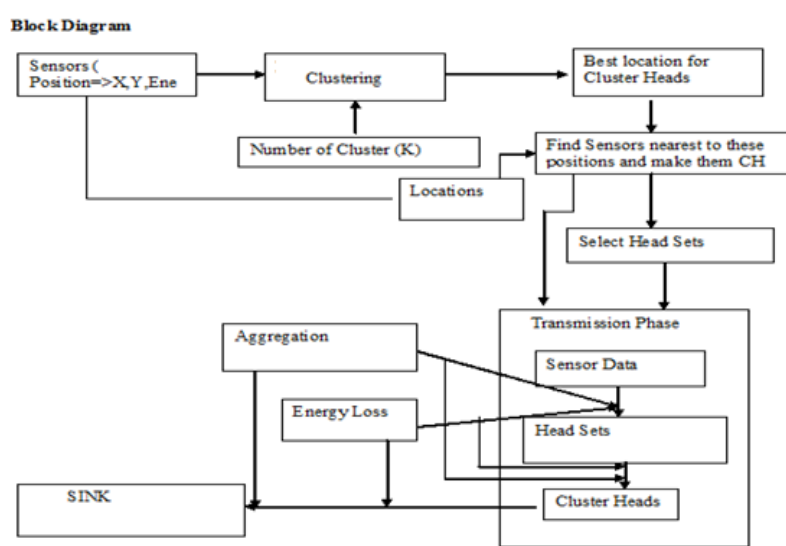

Figure 4.1 Basic Architecture of the proposed work

Figure 4.1 shows the basic architecture of the network. Firstly group of nodes, distributed over a geographic area select a cluster head depending upon the energy of the nodes. Once selected, this node acts as local data gathering and buffering node. It schedules all the cluster member's reception and is responsible for channel allocation. Once he data is gathered from the cluster members, it buffers the packets and forwards a single packet containing the aggregated data to the destination thereby reducing the overall number of packets being transmitted in the network. As and when this cluster head node starts loosing their energy the cluster members selects another node with higher energy from the group and the new node replaces the older cluster head as the cluster head node.

Finally the overall statistics of the simulation is gathered for analysis and comparison.

\subsection{MODULE DESCRIPTION}

\section{- Topology Creation}

To be able to run a simulation scenario, a network topology must first be created. In ns2, the topology consists of a collection of nodes and links.

\section{- $\quad$ Network Parameter Configuration}

The node arrangement in ns2 is a good task in which the number of nodes can be constructed for a set of parameters. The following table tells about the node configuration parameters.

\section{- $\quad$ Routing}

Routing is a process of determining systematically how to forward messages towards the destination node based on its address is called routing.

\section{- $\quad$ Packet Exchange}

- In networks, a packet is a box that transfers data over a TCP/IP network and internetworks.

- A packet normally represents the smallest amount of data that can go across the network at a single time.

- Packets are the basic objects for the data exchange.

- Ns2 has its own memory management for allocating packets.

\section{Performance monitoring}

- Performance monitoring means observe and check the progress or quality of (something) over a period of time.

\subsection{USE CASE DIAGRAM}

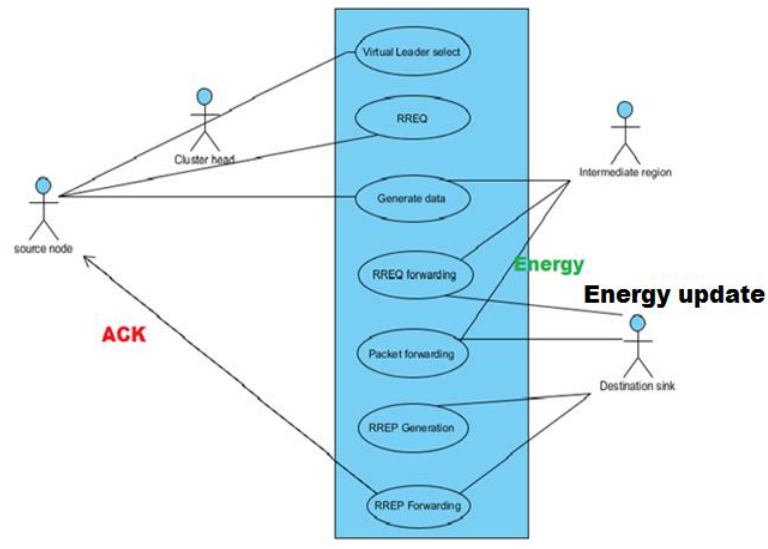

Figure 4.2 Use case Diagram

Description about use case diagram:

Steps

1. Firstly source node will create a cluster with cluster head.

2. Cluster head will select the virtual leader(one packet) in its group.

3. Next source node will send the Route request(RREQ) through intermediate region. And it will generate data.

4. Next intermediate will forward the RREQ and packet to destination with energy

5. After reaching packet to destination it will generate and forward the acknowledge through route reply(RREP).

\subsection{DATA FLOW DIAGRAM}

\section{0 level DFD}

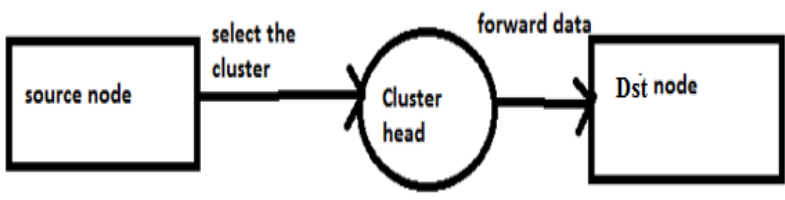

a) $0^{\text {th }}$ Level DFD 
INTERNATIONAL JOURNAL OF INNOVATIVE RESEARCH IN ELECTRICAL, ELECTRONICS, INSTRUMENTATION AND CONTROL ENGINEERING Vol. 3, Issue 12, December 2015

\section{1 level DFD}

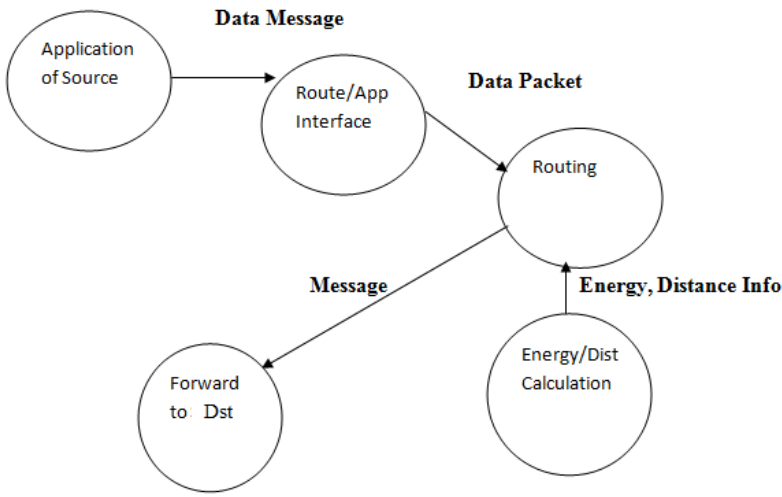

b) $1^{\text {st }}$ Level DFD

Figure 4.3 Data Flow Diagrams

Data flow diagram here presents the input output relationships through the core modules. As we can see that source nodes, the one which are transmitting and logging some physical parameters like energy transmit the aggregated data through the clusterhead nodes.

\subsection{EVENT SEQUENCE}

\subsubsection{SEQUENCE DIAGRAM}

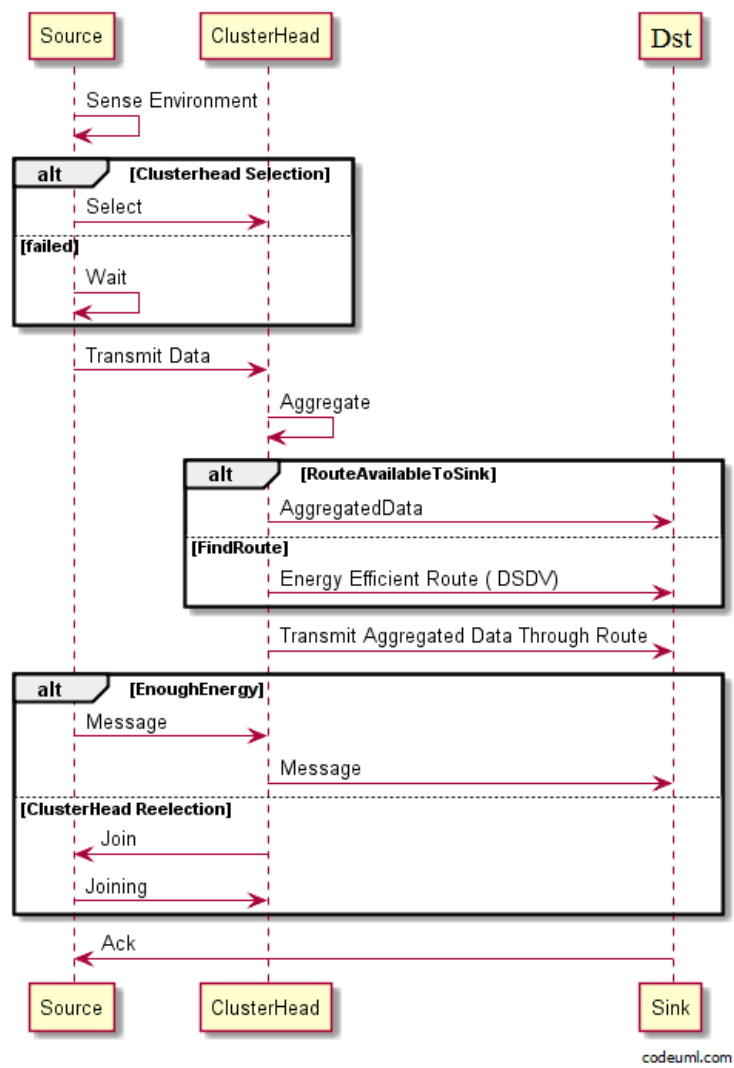

Figure 4.4 Sequence Diagram

Sequence diagram here presents the sequence of events takes place starting from transmitting to data transmission to the destination. Source mitigates the data to the cluster head. Cluster head waits for data from other sources. This data is then aggregated into a single packet. The packet is sent to the destination node. On the process both Clusterhead and the source looses energy. Because cluster head supports so many node, it's energy will dissipate faster. If clusterhead does not have enough energy, another cluster head will be selected dynamically among nodes having high energy.

\section{RESULTS}

\subsection{Graphs}

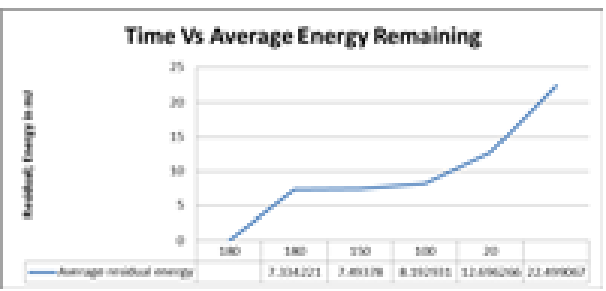

Figure 5.1: Simulation time Vs Average residual energy

The above graph shows that as the simulation time increases the nodes spent more and more energy. This is obvious fact in any networks. But in proposed system, the energy is balanced for intermediate amount of time. As time approaches to high value (180), energy of the nodes starts getting zero. Thus packet rate needs to be limited to prevent this event.

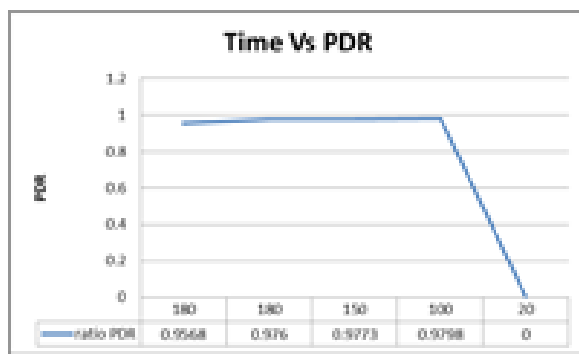

Figure 5.2: Simulation time Vs Packet Delivery Ratio

Packet delivery ratio increases with time. Once the network builds the clusterheads and routes, data transmission becomes $100 \%$. Thus the proposed design is verified.

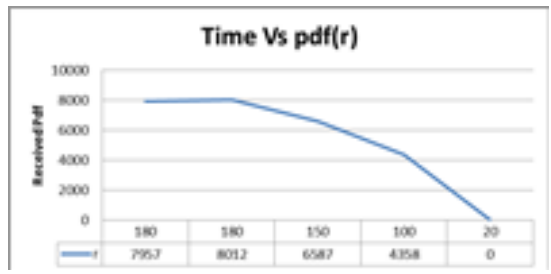

Figure 5.3: Simulation time Vs PDF (received)

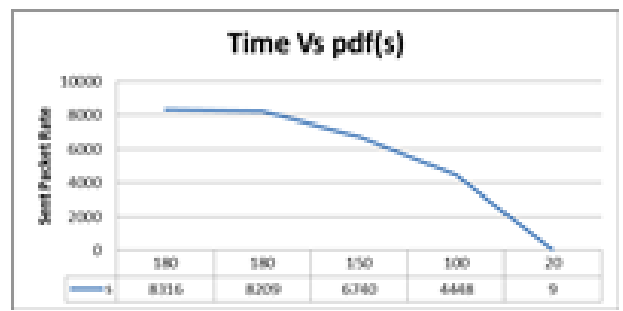

Figure 5.4: Simulation time Vs PDF (send)

Packet Delivery Function ( pdf) is a measure of generated traffic rate. The bove two graphs shows that the 
transmitted and received traffic builds up with cluster head But as the agricultural fields are large which needs long formation and route discovery and increases with time. Then after saturation limit, they get steady.

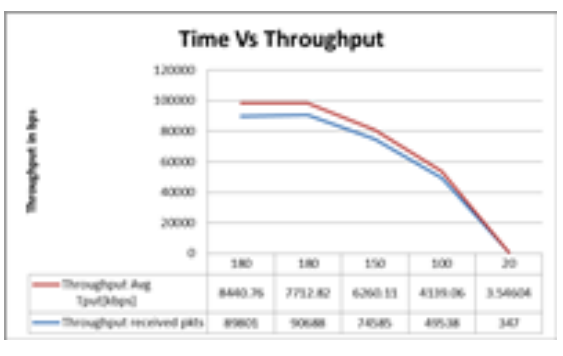

Figure 5.5: Simulation time Vs Throughput

As throughput is a direct function of Pdf, both overall throughput and received packet increases with time. But as simulation time is higher, received throughput comes down. This is because many nodes looses energy with time which results in many packet drop.

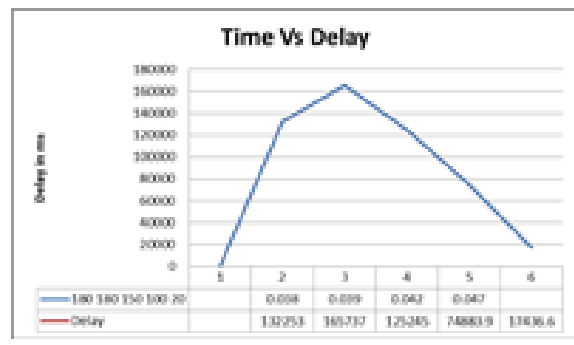

Figure 5.6: Simulation time Vs Delay

During intermediate phase of simulation, rate of transmission is very high, which results in high delay. As packet rate comes down, delay is also saturated.

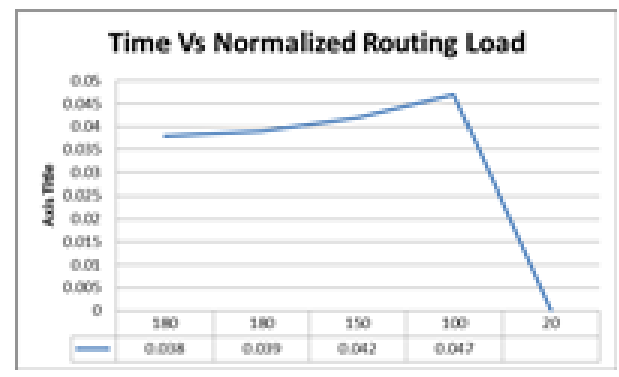

Figure 5.7: Simulation time Vs Normalized Routing Load

Normalized routing load gives the ratio of packet being forwarded by intermediate nodes divided by total packets. The graph shows that for most part of the simulation, all the node's load are balanced and this proves the proposed system

\section{CONCLUSION}

Mobile Adhoc network is exciting new area of study. With the ever demanding need for more food and ever reducing agro production, more technological paradigm shifts are desired. Adhoc Networks are breath of fresh air in many fields, but especially in agriculture. When Mobiles are placed at different parts of fields, they can remotely transmit the condition of the crop from remote areas. Thus monitoring cost is greatly decreased. range data transmission and range of the Mobiles are small, Mobiles are to be laid in high number. Mobiles are battery driven. When more transmission takes place, they looses their energy. It is not possible to manually replace each node's battery. Hence new techniques are needed that can help preserving energy in Adhoc network.

In this work we have presented energy preserving Adhoc network design with clusterheads. Clusterheads by aggregating in network data can significantly reduce the overall traffic

We have proved through simulation results that proposed system is variable and offers a good energy utilization. This work can be further improved by forming the clusterhead based on other parameters like link quality and changing the clusterheads in such a manner that no clusterheads are overloaded.

\section{REFERENCES}

[1] The network simulator - ns-2.33. http://www.isi.edu/nsnam/ns

[2] Douglas M. Blough, Mauro Leoncini, Giovanni Resta, and Paolo Santi. The k-neighbors approach to interference bounded and symmetric topology control in ad hoc networks. IEEE Transactions on Mobile Computing, 5(9):1267-1282, 2006.

[3] Benjie Chen, Kyle Jamieson, Hari Balakrishnan, and Robert Morris. Span: An energy-efficient coordination algorithm for topology maintenance in ad hoc wireless networks. ACM Wireless Networks, 8:85-96, September 2001.

[4] H. P. Gupta and S. V. Rao.Pclr: Power control-based locally customize routing for manets. In Proc. IEEE International Conference on RF and Signal Processing Systems, pages 632-637, 2010

[5] N. Li, J.C. Hou, and L. Sha. Design and analysis of an mst-based topology control algorithm. In INFOCOM 2003. Twenty-Second Annual Joint Conference of the IEEE Computer and Communications. IEEE Societies, pages 1702 - 1712, mar. 2003.

[6] Xiang-Yang Li and Peng-Jun Wan. Constructing minimum energy mobile wireless networks. SIGMOBILE Mobile. Computing. Communication. Rev.,5(4):55-67, 2001.

[7] A. Naghshegar A. Dana, A. Darehshoorzadeh and K. Karimpoor. Topology control scheme in Manetsfor AODV routing. In Information and Communication Technologies: From Theory to Applications, 2008.ICTTA 2008. 3rd International Conference on, pages 1-6, 7-11 2008.

[8] Charles E. Perkins and Elizabeth M. Royer. Ad-hoc on-demand distance vector routing. In WMCSA '99: Proceedings of the Second IEEE Workshop on Mobile Computer Systems and Applications, pages 90-100, Washington, DC, USA, 1999. IEEE Computer Society.

[9] Ram Ramanathan and Regina Hain. Topology control of multihop wireless networks using transmit power adjustment. In INFOCOM, pages 404-413, 2000.

[10] Jang-Ping Sheu, Shin-ChihTu, and Chi-Hung Hsu. Location-free topology control protocol in wireless ad hoc networks Computing. Communication.,31(14):3410-3419, 2008 